University of Massachusetts Amherst ScholarWorks@UMass Amherst

2001

\title{
Discovery of Molecular Gas in the Outflow and Tidal Arms around M82
}

CL Taylor

F Walter

Min Yun

University of Massachusetts - Amherst

Follow this and additional works at: https://scholarworks.umass.edu/astro_faculty_pubs Part of the Astrophysics and Astronomy Commons

\section{Recommended Citation}

Taylor, CL; Walter, F; and Yun, Min, "Discovery of Molecular Gas in the Outflow and Tidal Arms around M82" (2001). The Astrophysical Journal Letters. 1142.

$10.1086 / 338048$ 
Draft VERSION FEBRUARY 1, 2008

Preprint typeset using $\mathrm{LAT}_{\mathrm{E}} \mathrm{X}$ style emulateapj v. 25/04/01

\title{
DISCOVERY OF MOLECULAR GAS IN THE OUTFLOW AND TIDAL ARMS AROUND M82
}

\author{
Christopher L. TAYLOR \\ Five College Radio Astronomy Observatory, University of Massachusetts, 619 Lederle GRT, Amherst, MA \\ 01003 \\ FABIAN WALTER \\ California Institute of Technology, Astronomy Department 105-24, Pasadena, CA, 91125 \\ AND \\ MiN S. YUN \\ Department of Astronomy, University of Massachusetts, 619 Lederle GRT, Amherst, MA 01003 \\ Draft version February 1, 2008
}

\begin{abstract}
We present the first fully sampled map of ${ }^{12} \mathrm{CO}(1-0)$ emission from M82 covering the entire galaxy. Our map contains a $\sim 12 \times 15 \mathrm{kpc}^{2}$ area. We find that extraplanar $\mathrm{CO}$ emission, previously reported at short distances above the galactic plane, extends to heights of up to $6 \mathrm{kpc}$ above the disk. Some of this emission is associated with tidal arms seen in HI, implying either that M82 contained substantial amounts of molecular gas in the outer disk, or that molecular gas formed after the tidal features. CO emission along the direction of the outflow extends to distances of $\sim 3 \mathrm{kpc}$ above and below the disk. At this distance, the line is shifted in velocity $\sim 100 \mathrm{~km} \mathrm{~s}^{-1}$, and has the same sense as the galactic outflow from the central starburst. This implies that molecular gas may be entrained into the outflow.
\end{abstract}

Subject headings: Extragalactic Astronomy-ISM: molecules — Galaxies: individual(M82) — Galaxies: ISM — Galaxies: starburst — Radio lines: galaxies

\section{INTRODUCTION}

M82 is one of the closest starburst galaxies $(\mathrm{D}=3.63$ Mpc assuming the distance of M81; Freedman et al. (1994)), and one of the most intensively studied; it has been observed at nearly every wavelength possible (Lehnert et al. (1999) summarize the past observations). It is a well known prototype of the starburst-driven galactic superwind phenomenon (Axon \& Taylor 1978). The galactic winds, created by the combined effect of supernovae and stellar winds (Tenorio-Tagle \& Bodenheimer 1988), are thought to drive material up out of the disk. M82 is also a member of a triplet system (with M81 and NGC 3077) undergoing a strong gravitational interaction (Yun, Ho \& Lo 1994). Material can be drawn out of the disk by such interactions, creating tidal features up to tens of kiloparsecs in length (e.g. Smith, Struck \& Pogge (1997), Irwin (1994)). M82 features both a superwind and tidal arms.

M82 has been observed in various CO lines, both with single dish telescopes and interferometers (e.g. Olofsson \& Rydbeck (1984), Young \& Scoville (1984), Nakai et al. (1987), Sofue et al. (1992), Shen \& Lo (1995), Weiß et al. (1999), Weiß et al. (2001), and Seaquist \& Clark (2001)), mostly focusing on the inner $1 \mathrm{kpc}$ of the galaxy. In this paper we present the first fully sampled, wide-field map of the ${ }^{12} \mathrm{CO} \mathrm{J}=1 \rightarrow 0$ emission in M82, covering an area of $\sim 12 \times 15 \mathrm{kpc}$ (improving previous areal coverage by more than an order of magnitude) to search for molecular gas associated with either the superwind or the tidal features.

\section{OBSERVATIONS AND DATA REDUCTION}

The observations were obtained with the Five College Radio Astronomy Observatory $14 \mathrm{~m}$ telescope over several runs from January - May, 2001. We observed the ${ }^{12} \mathrm{CO} \mathrm{J}=1 \rightarrow 0$ line using SEQUOIA, a focal plane array receiver, consisting of 16 pixels. Because the beamsize of the telescope at $115 \mathrm{GHz}$ is $44^{\prime \prime}$ and the spacing between pixels in the array is $88^{\prime \prime}$, the beams were shifted in halfbeam steps to fill in the gaps and make a fully sampled map. The extragalactic filterbanks were used to obtain a bandwidth of $320 \mathrm{MHz}$ with $5 \mathrm{MHz}\left(13 \mathrm{~km} \mathrm{~s}^{-1}\right)$ channels. System temperatures ranged from $\sim 450$ to $800 \mathrm{~K}$. The pointing was checked every 2 to 4 hours each session using $\mathrm{SiO}$ maser sources.

Six SEQUOIA footprints $\left(6^{\prime} \times 6^{\prime}\right)$ were observed, partly overlapping, for a total area of $12^{\prime} \times 15^{\prime}$ (Fig. 2). The data were reduced with the CLASS package. We subtracted linear baselines from each spectrum and when a good fit didn't result, the spectrum was discarded. Over 9,000 spectra were collected and coadded; the total integration time on source was $\sim 45$ hours. A main beam efficiency of $\eta_{M B}=0.45$ was used used to put the spectra on the $\mathrm{T}_{M B}$ scale. We smoothed the data spatially to $90^{\prime \prime}$ and in velocity to $39 \mathrm{~km} \mathrm{~s}^{-1}$ to emphasize low $\mathrm{S} / \mathrm{N}$ extended structure. Unless otherwise stated, all discussion refers to the smoothed data cube.

\section{ANALYSIS AND RESULTS}

\subsection{The Global Distribution of the Gas}

Our observations reveal molecular gas at large heights above and below the plane of M82: Fig. 1 shows the channel maps, and Fig. 2 the total integrated $\mathrm{CO}$ emission. Given the strong emission from the center of M82 (0.76 $\mathrm{K}$ in our $60^{\prime \prime}, 39 \mathrm{kms}^{-1}$ data), it is possible that any weak emission might be the response of the telescope error beam to the strong source. A careful inspection of our data shows this isn't a problem in our map for two reasons: 1 ) the velocity of the extended emission is shifted relative to the center of M82 (Fig. 6), and 2) the response of the error beam would be 100 to 1000 times weaker than the peak emission, but the peak of the extended emission is 


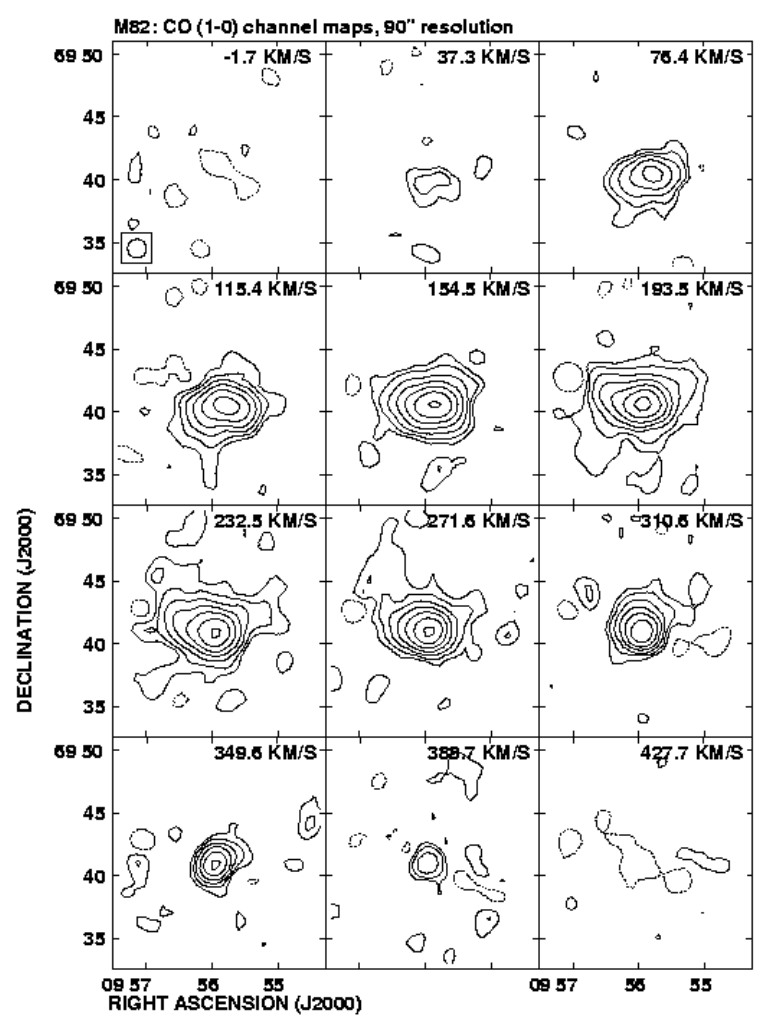

FIG. 1.- Channel maps showing ${ }^{12} \mathrm{CO}$ (1-0) emission,in M82 smoothed to a resolution of $90^{\prime \prime}$. The contours are in units of $-2,2$, $4,8,16,32,64$ and $128 \sigma$ where $\sigma$ is the rms noise in a channel map, equal to $6.6 \mathrm{mK}$.

only about 15 times weaker.

North of the galaxy, molecular gas is found up to $6 \mathrm{kpc}$ (all values are deconvolved for the 90" beam, $1^{\prime} \sim 1 \mathrm{kpc}$ for the adopted distance of $3.63 \mathrm{Mpc}$ ) away from the plane of the disk. More emission is detected $\sim 4 \mathrm{kpc}$ above the disk at $\sim 2 \mathrm{kpc}$ west of the minor axis. Along the minor axis, emission reaches heights of $\pm \sim 3 \mathrm{kpc}$. The two features seen towards the south are probably not real (see the discussion below).

The total CO integrated intensity is $6.1 \times 10^{2} \mathrm{~K} \mathrm{~km} \mathrm{~s}^{-1}$. Assuming a Galactic CO-to- $\mathrm{H}_{2}$ conversion factor of $2.3 \times 10^{20}$ $\mathrm{cm}^{-2}\left(\mathrm{~K} \mathrm{~km} \mathrm{~s}^{-1}\right)^{-1}$ (Strong et al. 1988) yields $4.3 \times 10^{9}$ $\mathrm{M}_{\odot}$ (but see (Weiß et al. 2001) for a lower conversion factor in M82). This compares to the value of $1.7 \times 10^{9}$ $\mathrm{M}_{\odot}$ obtained by (Young \& Scoville 1984) (after correcting to our assumed distance and conversion factor). The difference between our value and theirs results from several factors: 1) they did not convert to $\mathrm{T}_{m b}$ so with an $\eta_{M B}$ of 0.5 at that time, the two values agree to within $20 \%$; 2) they didn't map the entire galaxy; and 3) they had a sparse sampling away from the galactic disk.

To determine how much emission originates outside the disk of M82, we measured the FWHM of the emission along the minor axis $\left(\sim 120^{\prime \prime}\right)$ and defined the extraplanar emission to be that which originates at a distance of at least $120^{\prime \prime}$ from the major axis. This flux adds up to $10 \%$ of the total. Because our large beam doesn't resolve

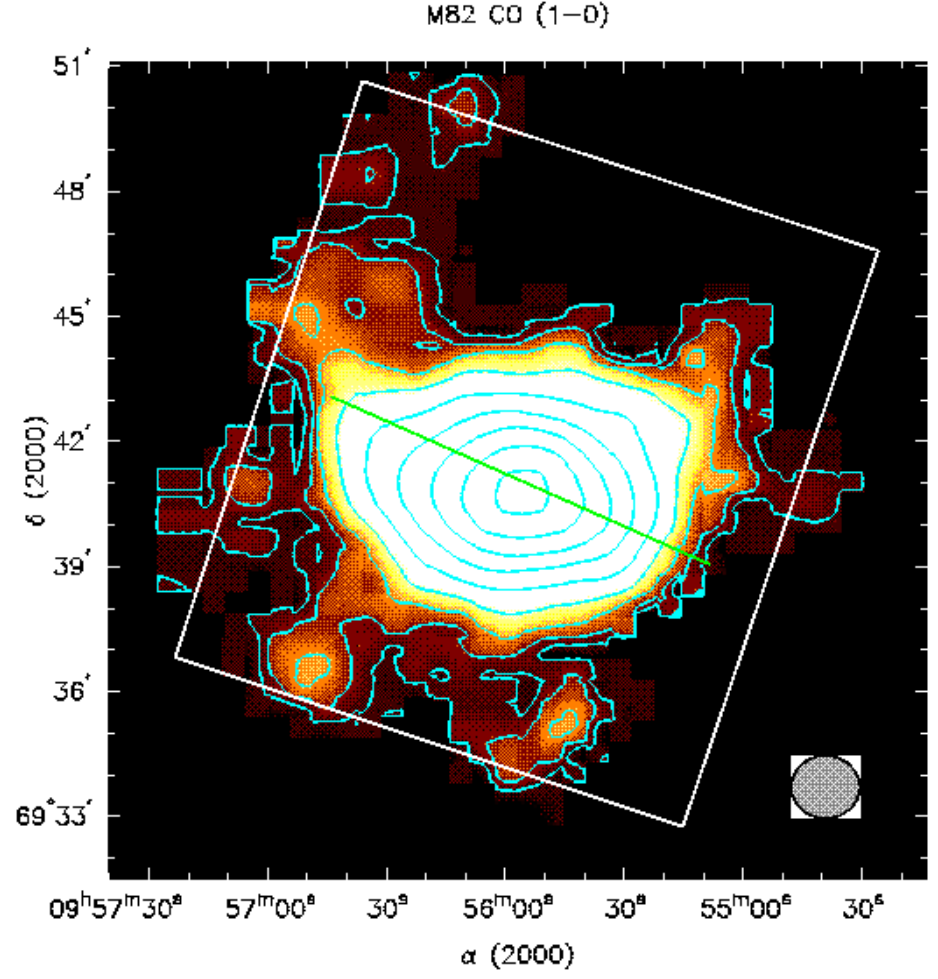

FIG. 2.- An integrated intensity image and contours of ${ }^{12} \mathrm{CO}(1-$ 0 ) emission in M82, smoothed to $90^{\prime \prime}$ resolution. The data cube was blanked at the $2 \sigma$ level prior to making this image. The contours are $0.31,0.63,1.25,2.5,5,10,20,40$ and $80 \%$ of the peak value $\left(1.0 \times 10^{5} \mathrm{~K} \mathrm{~km} \mathrm{~s}^{-1}\right.$ beam $^{-1}$. The green line shows the plane of the galaxy disk. The beam size is indicated in the lower right. The box shows the region mapped, where emission contours extend beyond the box because we regridded and spatially smoothed the data.

the disk emission, it is hard to decompose this properly, so this number should be taken with caution.

\subsection{Kinematics}

Some of the CO emission is clearly associated with tidal features. Fig. 3 shows the CO (1-0) contours superposed over an HI map. The HI map combines unpublished B, C \& D configuration data from the NRAO-VLA ${ }^{1}$ with a $21^{\prime \prime}$ $\times 19^{\prime \prime}$ beam. The HI arm projecting off the northeast (NE) end is the most obvious tidal feature, and the molecular gas here is associated with this arm. To the northwest (NW) of the galaxy, the CO emission closely follows a set of HI clouds. HI and $\mathrm{CO}$ spectra for both regions (NE and NW) are presented in Fig. 4. The HI is not situated along the axis of outflow, so both the $\mathrm{CO}$ and $\mathrm{HI}$ emission are probably of tidal origin. The NW feature lies closer to the minor axis of M82 and may be influenced by the starburst driven outflow. In the south (S1) the $\mathrm{CO}$ contours seem to follow the HI closely in the spur projecting more than $300^{\prime \prime}$ southeast of the galaxy center. However, as Fig. 4 shows, the velocities of the HI and CO don't match here. The cloud to the very south $(\mathrm{S} 2)$ has no corresponding HI emission at any velocity. These two southern features (and the eastern edge of NE) fall within one $90^{\prime \prime}$ beam of our map edge. Because there is no data beyond the map edge, smoothing spatially will increase the noise along the edge,

1 The National Radio Astronomy Observatory is a facility of the National Science Foundation operated under cooperative agreement by Associated Universities, Inc 


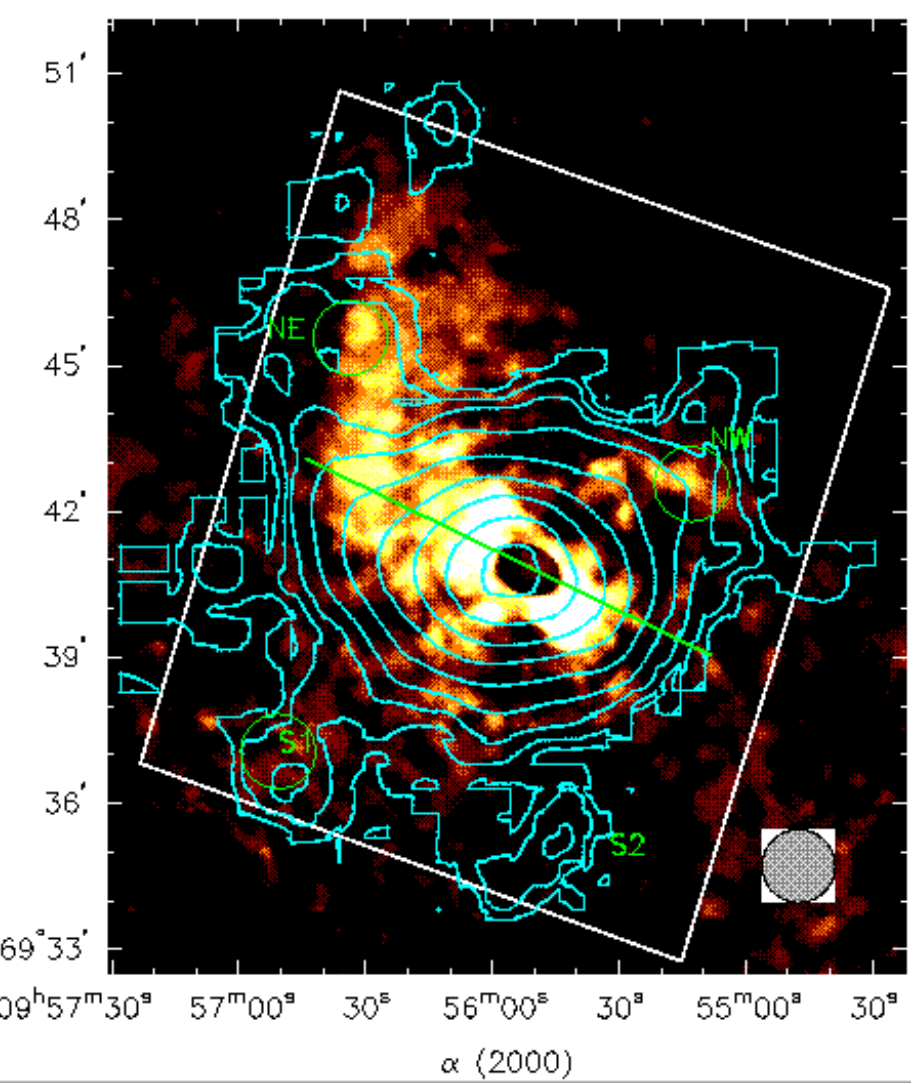

FIG. 3.- ${ }^{12} \mathrm{CO}(1-0)$ contours superposed on a VLA HI integrated intensity map. The $\mathrm{CO}$ contours are the same as in Fig. 2, and the $\mathrm{CO}$ beam size is shown in the lower right. The white box indicates the region mapped in $\mathrm{CO}$. The green line indicates the major axis of M82. The circles show the areas where the $\mathrm{CO}$ and HI spectra are compared in Fig. 4.

relative to the interior of the map; these edge features may be artifacts.

While some of the $\mathrm{CO}$ emission away from the disk is of tidal origin, some participates in the well known outflow in M82. Previous observations found that CO near the major axis is outflowing $\left( \pm 42^{\prime \prime}\right.$ in Seaquist \& Clark (2001); $\pm 45^{\prime \prime}$ in Nakai et al. (1987) ), but we show for the first time that the molecular outflow extends to $\sim 3$ kpc $\left(180^{\prime \prime}\right)$. Fig. 5 shows the CO contours superposed on a continuum subtracted $\mathrm{H} \alpha$ image obtained from the Astronomical Digital Image Library (http://adil.ncsa.uiuc.edu/ ). Excluding the tidal features, the shape of the $\mathrm{CO}$ contours is similar to the outflow traced in $\mathrm{H} \alpha$. However, due to our low spatial resolution we cannot correlate the $\mathrm{CO}$ emission to specific $\mathrm{H} \alpha$-filaments. In any event, it is not clear that a correlation is expected as the molecules would be dissociated before the gas is ionized.

Fig. 6 shows evidence that molecular gas is affected by the galactic outflow. We show three spectra from along the minor axis of M82 - north (N) and south (S) of center, and at the center (C) (indicated in Fig. 5). The offset in velocity between the $\mathrm{S}$ and $\mathrm{C}$ spectra is obvious and amounts to a difference of $100 \mathrm{~km} \mathrm{~s}^{-1}$. The $\mathrm{N}$ spectrum is very wide, however, and lacks a central peak, making it hard to discern an offset compared to the galaxy center. It appears to contain not only emission from the outflow

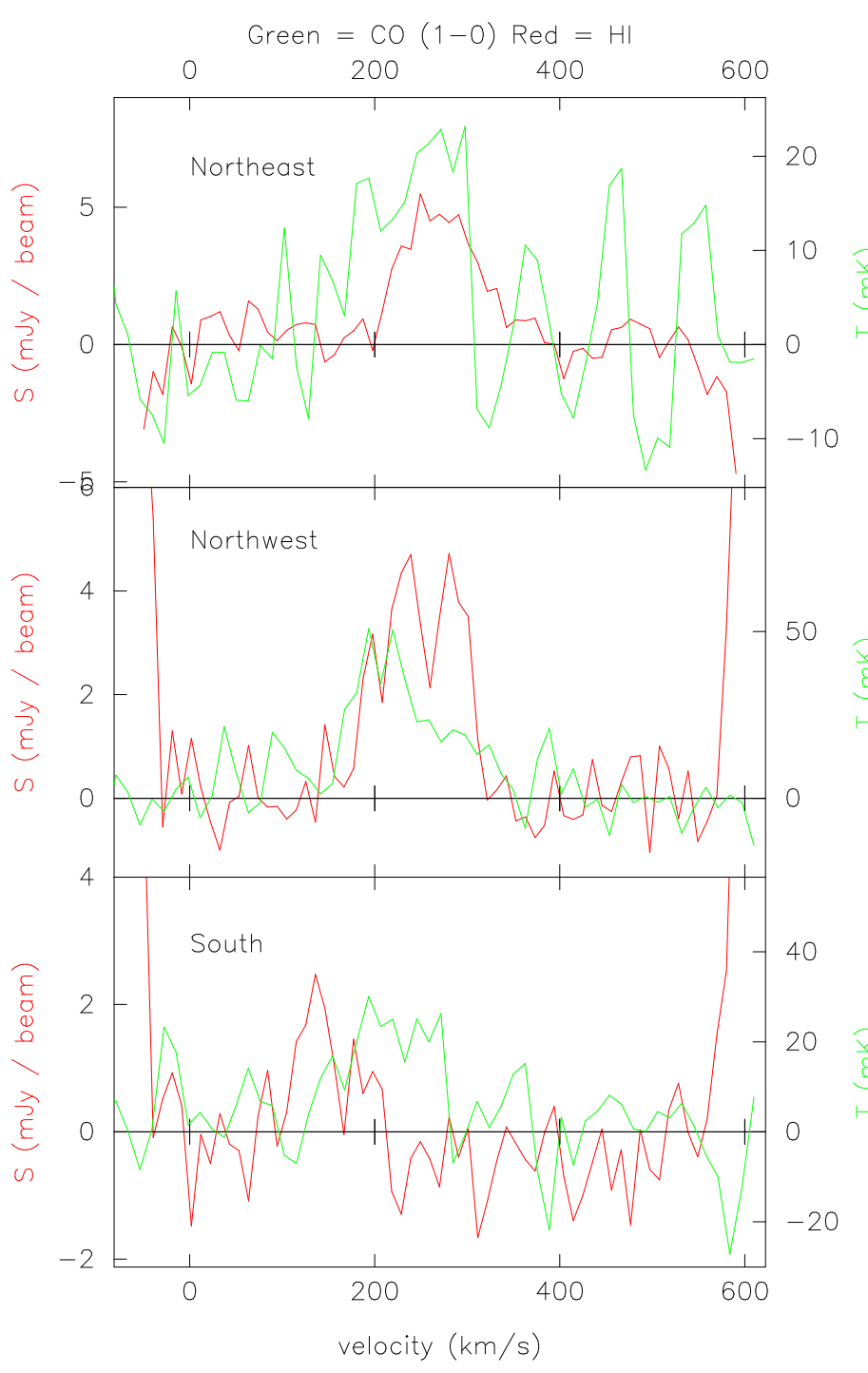

FIG. 4.- Spectra through the $\mathrm{CO}$ and HI data cubes for the three tidal features in the text. The $\mathrm{HI}$ is plotted in red, the $\mathrm{CO}$ in green.

(at $\sim 300 \mathrm{~km} \mathrm{~s}^{-1}$ ), but contamination from the northwest tidal feature $(\mathrm{TF})$. We plot in red the TF spectrum over the emission from $\mathrm{N}$, showing that the emission at lower velocities at $\mathrm{N}$ is similar in velocity range to that from $\mathrm{TF}$. Obtaining an outflow velocity here is problematic; due to the contamination from $\mathrm{TF}$ it is impossible to isolate the minor axis emission. If we assume the minor axis outflow contribution dominates at the extreme redshifted range of velocities, then we obtain a value of $\sim 80 \mathrm{~km} \mathrm{~s}^{-1}$, but this should be taken with a grain of salt. The redshift of $\mathrm{S}$ and possible blueshift of $\mathrm{N}$ have the same sense as observed in other lines (e.g. CO (3-2) by Seaquist \& Clark (2001); $\mathrm{H} \alpha$ by McKeith et al. (1995); Shopbell \& BlandHawthorn (1998)). This is generally attributed to a large scale outflow with an inclination of $80^{\circ}$ to the disk.

\section{SUMMARY}

We present the first complete, fully sampled map of the ${ }^{12} \mathrm{CO}(1-0)$ emission in the prototypical starburst galaxy M82, covering an area of $\sim 12 \times 15 \mathrm{kpc}^{2}$. M82 is nearly edge on $\left(i=80^{\circ}\right)$ allowing us to search for emission above the disk; we detect $\mathrm{CO}$ to great heights out of the galactic 
M82 Color: H-alpha, Contours: CO (1-0)

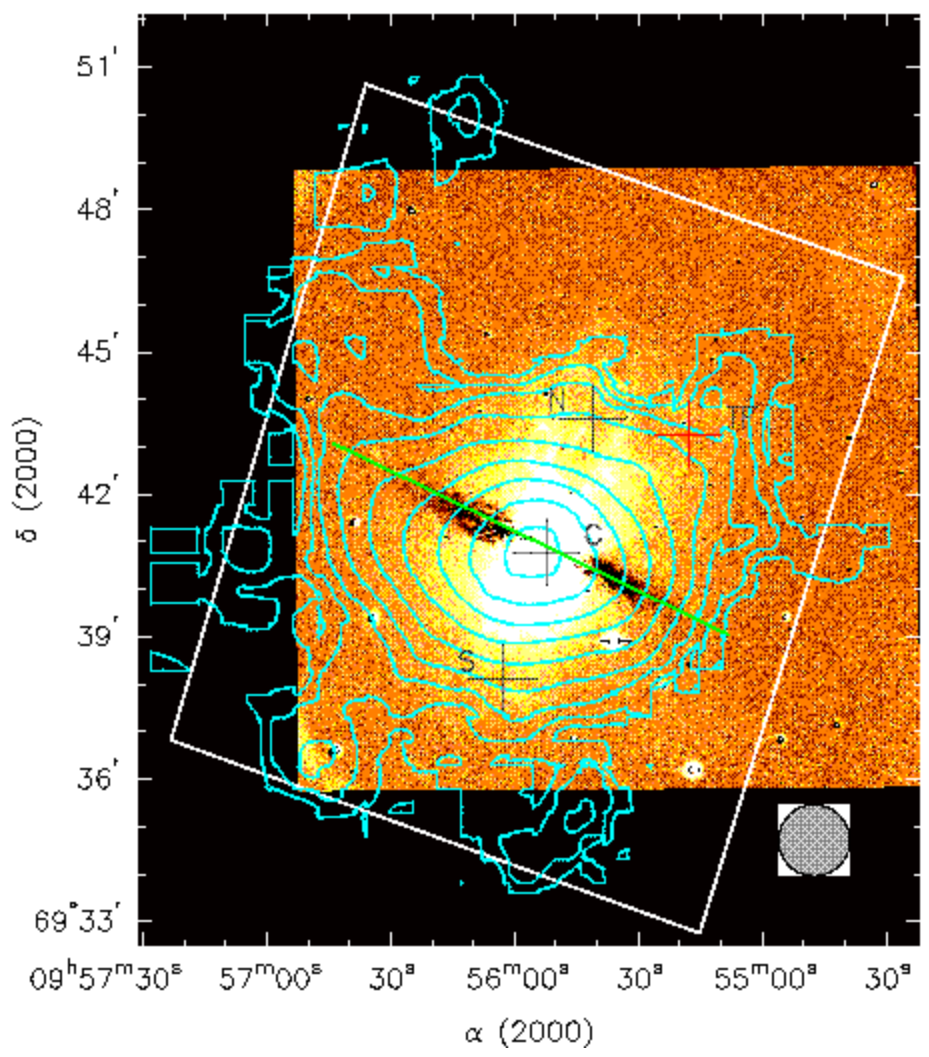

FIG. 5.- ${ }^{12} \mathrm{CO}$ (1-0) contours superposed on a continuum subtracted $\mathrm{H} \alpha$ map. The $\mathrm{CO}$ contours are from Fig. 2. The $\mathrm{CO}$ beam size is shown in the lower right. The green line indicates the major axis of M82. The crosses show the areas compared in Fig. 6

plane (up to $6 \mathrm{kpc}$ ). Some of this extraplanar molecular gas is in tidal arms generated by the interaction with M81. Gas in tidal arms normally comes from the outer disk regions, so that we see CO emission in the arms means that M82 either had molecular gas in its outer disk, or else the molecular gas formed in the tidal arms after the interaction. Either way, it also requires sufficient metal abundance to form the observed $\mathrm{CO}$ in addition to the implied $\mathrm{H}_{2}$. We see emission along the minor axis $\sim 3 \mathrm{kpc}$ above and below the disk. In the north some of this emission may be associated with tidal features, but in the south it is not. The velocity difference of the $\mathrm{CO}$ emission along the minor axis has the same direction as what is seen in the $\mathrm{H} \alpha$ emission line. This $\mathrm{CO}$ emission likely comes from molecular gas which has been entrained in the starburst driven outflow in M82.

We thank the referee for helpful comments that improved this paper. The Five College Radio Astronomy Observatory is operated with the permission of the Metropolitan District Commission, Commonwealth of Massachusetts, and with the support of the National Science Foundation under grant AST-9725951. FW acknowledges NSF grant AST 96-13717.

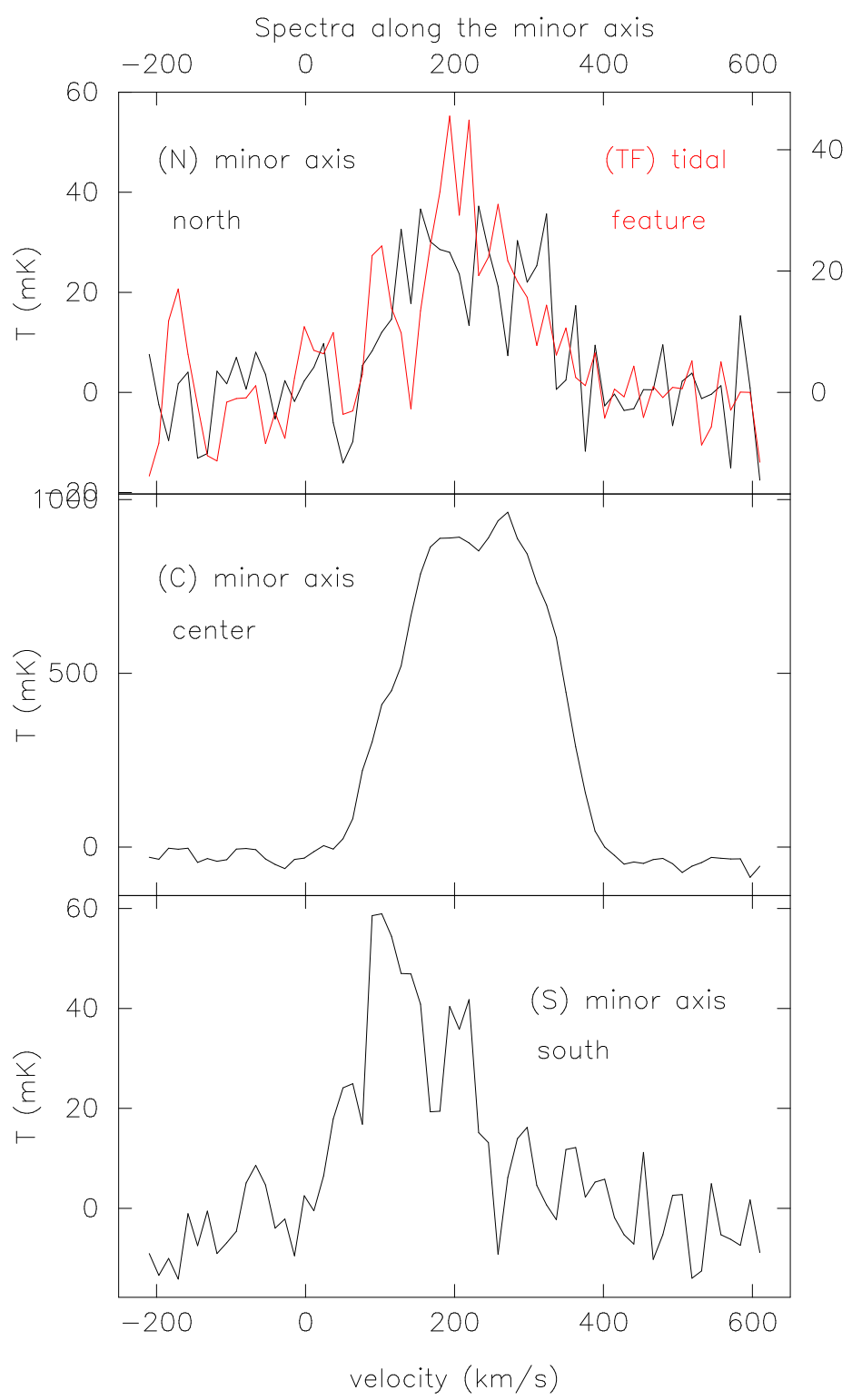

Fig. 6. - Spectra taken from positions along the minor axis. Bottom: the southern end of the minor axis. Center: the center of the galaxy. Top: the northern end of the minor axis, with a spectrum from the northwest tidal feature plotted in red for comparison.

Freedman, W.L. et al. 1994, ApJ, 427, 628

Irwin, J.A. 1994, ApJ, 429, 618

Lehnert, M.D., Heckman, T.M. \& Weaver, K.A. 1999, ApJ, 523, 575

McKeith, C.D., Greve, A., Downes, D. \& Prada, F. 1995, A\&A, 293, 703

Nakai, N., Hayashi, M., Handa, T., \& Saski, M. 1987, PASJ, 39, 685

Olofsson, H. \& Rydbeck, G. 1984, A\&A, 136, 17

Seaquist, E.R. \& Clark, J. 2001, ApJ, 552, 133

Shen, J. \& Lo, K.Y. 1995, ApJ, 445, L99

Shopbell, P.L. \& Bland-Hawthorn, J. 1998, ApJ, 493, 129

Smith, B.J., Struck, C. \& Pogge, R.W. 1997, ApJ, 483, 754

Sofue, Y., Reuter, H.-P., Krause, M., Wielebinski, R. \& Nakai, N. 1992, ApJ, 395, 126

Strong, A.W. et al. 1988, A\&A, 207,

Tenorio-Tagle, G. \& Bodenheimer, P. 1988, ARAA, 26, 145

Weiß, A., Neininger, N., Hüttemeister, S. \& Klein, U., 2001, A\&A, 365,571

Weiß, A., Walter, F., Neininger, N., \& Klein, U. 1999, A\&A, 345, L23

Young, J.S. \& Scoville, N.Z. 1984, ApJ, 287, 153

Yun, M.S., Ho, P.T.P., \& Lo, K.Y. 1994, Nature, 372, 530 